\title{
Design of the Electromagnetic - Piezoelectric Composite Vibration Energy Harvesting System
}

\author{
Sang Yingjun ${ }^{1 *}$, Li Man ${ }^{1}$, Wu Shangguang ${ }^{1}$, Cao Yang ${ }^{1}$, Huang Fei ${ }^{1}$, \\ Fan Yuanyuan ${ }^{2}$, Hao Yunrong ${ }^{2}$ \\ ${ }^{1}$ College of automation, Huaiyin Institute of Technology, Huaian, Jiangsu, China 223003 \\ ${ }^{2}$ College of mathematics and physics, Huaiyin Institute of Technology, Huaian, Jiangsu, China \\ 223003 \\ Email:sangyingj@163.com
}

\begin{abstract}
Keywords: Piezoelectric; Electromagnetic; Energy harvesting; PSpice simulation; Circuit design Abstract: In recent years, with the rapid development of micro electromechanical systems, low-power sensor networks and integrated circuit technology, the power supply problems are becoming the barrier of environment protection in practical and industrial. Vibration is a widely used energy in the environment, which has the potential to power the low power consumption system. However, the traditional vibration energy harvesting structure is relatively simple and its power generation capacity is weak, so we propose an electromagnetic and piezoelectric composite energy harvesting system based on cantilever structure. An composite energy conversion circuits is designed to transform the alternating current (AC) to direct current (DC), and the experiment verified the feasibility of the circuit, and the total power is increased than single mode.
\end{abstract}

\section{Introduction}

With the fast development of micro electromechanical systems, low-power sensor and integrated circuit technology, the electronic devices are being miniaturized, the power consumption of the micro circuit is becoming smaller and smaller, and the application of MEMS products and wireless sensor networks is becoming more and more popular[1]. At present, battery is the main way of energy supply of the most wireless sensor nodes, however, wireless sensors are hard to make full use for the traditional battery's shortcoming on energy Limited, regular replacement, pollution of the environment and so on, thus it is becoming a hot research topic how to design a energy harvesting device which turns the variation of the external environment into energy. Nowadays, the modes of the vibration generator include electrostatic, magnetostrictive, electromagnetic, piezoelectric and composite mode, which harvest energy at the same time to improve system power generation performance [2].

In this paper, an electromagnetic - piezoelectric composite vibration energy collection device is introduced, and a feasible energy conversion and storage circuit is designed.

\section{System principle and mathmatic model}

System structure principle. In this paper, a new type of piezoelectric- electromagnetic composite electric power generation device is studied which is based on the cantilever beam structure in this paper. The composite power generating device consists of an permanent magnet at the end of the cantilever instead of a mass block and the induction coils which is fixed bellow. The structure diagram is shown in Fig. 1.

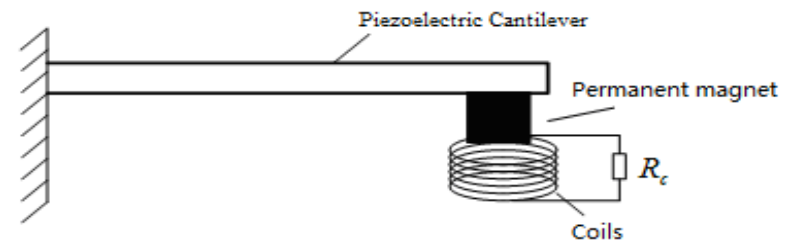

Fig.1 The schematic of the electromagnetic- piezoelectric energy harvesting 
The principle of the electromagnetic - piezoelectric composite energy harvester is that the induced electromotive force is produced by the relative motion of the permanent magnet and the coils when the cantilever beam is vibrated. The cantilever resonance frequency can be adjusted observably with the adjustment of the size of the permanent magnet mass, and it is adjusted close to the environmental vibration frequency to improve the efficiency of power generation.

The System Mathematical Model. According to the schematic above, the electromagnetic piezoelectric composite energy harvester is compounded by the collection of energy separately of electromagnetic and piezoelectric.

The Mathematical Model of Electromagnetic element. The basic principle of the electromagnetic power is the Ferrari's law of electromagnetic induction. The Ferrari's law of electromagnetic induction can be expressed as:

$$
\varepsilon=-N \frac{d \varphi}{d x}=-N \frac{d}{d t} \int B \cdot d S
$$

Where $\varepsilon$ represents EMF, $B$ represents electromagnetic induction intensity, $\mathrm{N}$ represents turns of coils of the closed loop, $\varphi$ represents the magnetic flux of through each turn coil, $S$ represents the area vector of coils. The electromagnetic power can be equivalent to a current source in parallel with the resistance. The equivalent circuit is shown as Fig.2.

The Mathematical model of piezoelectric element. The principle of piezoelectric power generation device is mainly based on the piezoelectric effect of piezoelectric material. When the piezoelectric materials subject to external stress, it will polarize in the internal and generate charge on the surface. When T represents stress and E represents electric field strength, which are seen as the independent variable, piezoelectric equation can be expressed in the way of tensor components as:

$$
S_{i}=S_{i u}^{E} T u+d_{j i} E_{j}
$$

In the formula $S_{i u}^{E} T u$ is the strain produced by stress when the electric field strength E is zero; ${ }_{j i} E_{j}$ is the strain under the action of electric field strength. The resistance of the piezoelectric sheet is too small to be ignored, so the piezoelectric power generation can be equivalent to a current source in parallel with a capacitor. ${ }^{C p}$ represents the inner electrode capacitance of the piezoelectric sheet. The equivalent circuit is shown as Fig.3.

The Model of System Output Power. In this design, the typical spring - mass - damper system is introduced, which is shown in Fig.4. The mass $m_{s}$ and the spring stiffness $k_{s}$ represent the generalized mass and the generalized stiffness of the vibration structure separately. In this model, damping is divided into mechanical damping $b_{m}$ and electrical damping $b_{e} . b_{m}$ represents the energy loss at the result of the structure and resistance factor, while $b_{e}$ represents the captured energy coming from the different energy harvesting principle, which can be divided into piezoelectric damping ${ }^{b_{e p}}$ and electromagnetic damping $b_{e m}$, so the total system damping is: $b_{t}=b_{m}+b_{e p}+b_{e m}$.



Fig. 2 The equivalent circuit of the electromagnetic generation sources

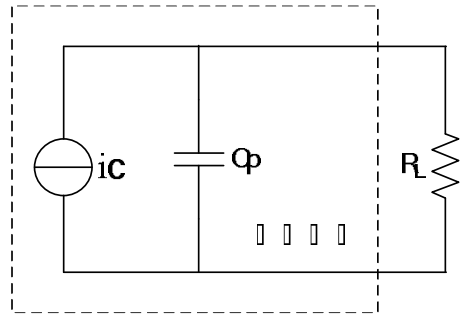

Fig. 3 The equivalent circuit of the piezoelectric generator power



Fig.4 The vibration model of the composite energy harvesting system

$y(t)=Y_{0} \sin \omega t$ is a sine excitation which is excited by the external environment, $z(\mathrm{t})$ represents the displacement that the mass $\mathrm{m}_{\mathrm{s}}$ relative to the entire system. The differential equation of motion for the system is: 


$$
m_{s} \ddot{z}(t)+b_{t} \dot{z}(t)+k_{s} z(t)=-m_{s} \ddot{y}(t)
$$

The inertia $F=-m_{s} \ddot{y}(t)$ acting on the mass is equal to the inertia acting on the whole system[3]. The system instantaneous power can be expressed as:

$$
p(t)=-m_{s} \ddot{y}(t)[\dot{y}(t)+\dot{z}(t)]
$$

The systemic output energy from the formula can be expressed as:

$$
p_{c o}=\frac{m_{s}\left(\frac{\omega}{\omega_{n}}\right)^{3} \omega^{3} \zeta_{t} Y_{0}{ }^{2}}{\left[1-\left(\frac{\omega}{\omega_{n}}\right)^{2}\right]^{2}+\left(2 \zeta_{t} \frac{\omega}{\omega_{n}}\right)^{2}}
$$

In the formula $\omega$ represents the vibration frequency of the harmonic oscillator; $\omega_{n}$ represents the system undamped resonant frequency, $\omega_{n}=\sqrt{k_{s} / m_{s}} ; \zeta_{t}$ represents the total damping ratio, $\zeta_{t}=\zeta_{m}+\zeta_{e p+}+\zeta_{e m} ; \xi_{m}$ represents the mechanical damping ratio, $\zeta_{m}=b_{m} / 2 m_{s} \omega_{n}$, and $\zeta_{e p}, \zeta_{c m}$ represents the electrical damping ratio, $\zeta e i=b_{e i} / 2 m_{s} \omega_{n}$.

The output power is biggest when the system operates in a resonant state[4], $\omega=\omega_{n}$. At this time, the output energy can be expressed as:

$$
p_{c o}=\frac{m_{s} \omega_{n}{ }^{3} Y_{0}{ }^{2}}{4 \zeta_{t}{ }^{2}}
$$

\section{The energy harvesting circuit Simulation Design}

In the output power model, spring - mass - damper system model is adapted to describe the performance of energy harvesting system in this design. However, the model is an abstract model, and the effect of some parameters is ignored, so the electromagnetic - piezoelectric composite energy harvesting circuit is designed in this section, and PSpice is used to analyze the circuit. Based on the characteristic and analysis of the vibration generation micro power, the DC-DC converter circuit based on the IC chip is designed, which supplies to the load through the rectification, energy storage and pressure regulating. When the $\mathrm{AC}$ generated by the power generation device passes through a rectifier bridge, the electric energy is stored in a super capacitor, which can power the load directly after the DC passes through the regulated voltage chip.
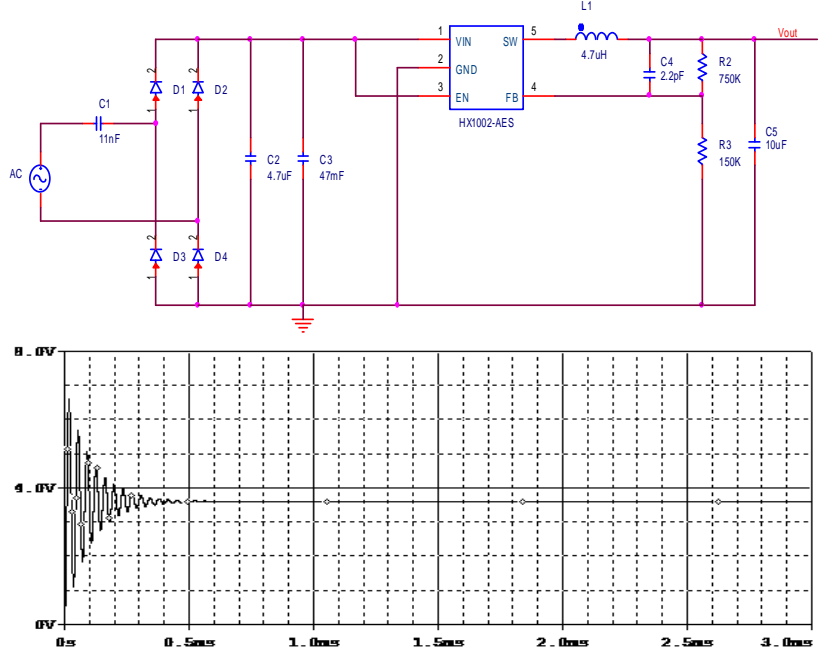

Fig.5 The piezoelectric element output simulation
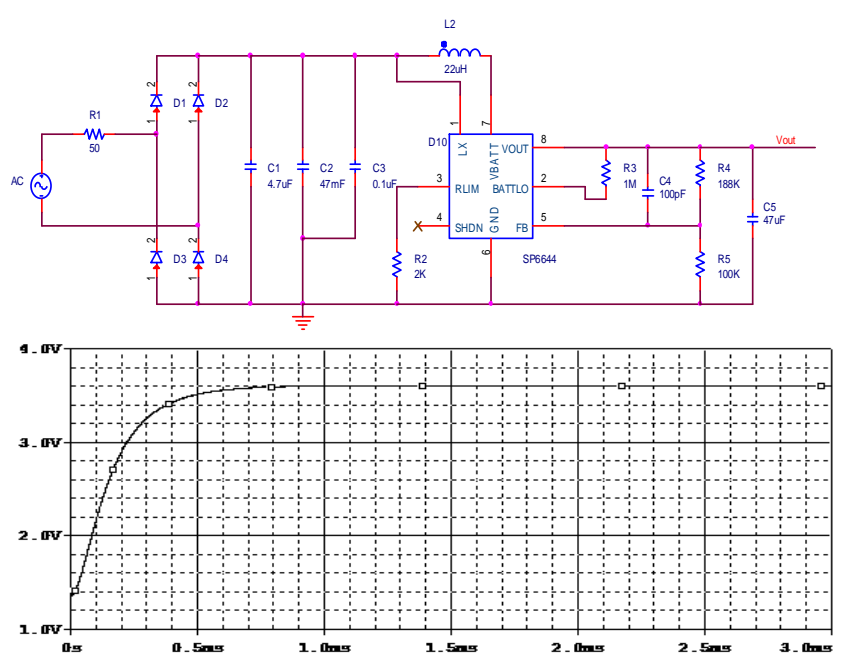

Fig.6 The electromagnetic unit output simulation

According to the piezoelectric equivalent circuit, the power supply is approximately equivalent to a sinusoidal voltage source $\mathrm{V} 1(5 \mathrm{~V}, 12 \mathrm{~Hz})$ series with a electrode capacitance $\mathrm{C}_{2}$. The piezoelectric chip capacitor is determined to $22 \mathrm{nF}$ temporarily. When the resistance is $480 \mathrm{k} \Omega$, the piezoelectric power supply is close to the maximum output power, which is about $18.1 \mathrm{uW}$. We can obtain the 
high-quality DC voltage of 3.6V smoothing by the HX1002 chip, the circuit and output DC voltage is shown in the Fig.5.

According to the electromagnetic equivalent circuit, the power supply is approximately equivalent to a sinusoidal voltage source V1 $(3 \mathrm{~V}, 12 \mathrm{~Hz})$ series with an electrode capacitance $\mathrm{C}_{2}$. The resistance of the coil is $1.47 \mathrm{k} \Omega$. Through the simulation, the electromagnetic power supply can output the DC voltage of about $2.6 \mathrm{~V}$ after rectification at the open circuit state. When the resistance of the load is $1.8 \mathrm{k} \Omega$, the electromagnetic power supply is close to the maximum output power about $3.1 \mathrm{~mW}$. The output DC voltage from the SP6644 chip is about 3.6V as shown in Fig.6.

\section{The Design of Experiment}

The printed PCB circuit is shown in Fig.7, and the breadboard is shown in Fig.8. The external vibration source exited the device, and the piezoelectric element at the open circuit state can generate the peak voltage of $5.4 \mathrm{~V}$, while the electromagnetic element can generate the peak voltage of $2.5 \mathrm{~V}$. The DC voltage of the circuit output in the piezoelectric circuit mode, the electromagnetic circuit mode and the composite mode is $3.1 \mathrm{~V}, 3.6 \mathrm{~V}$ and $2.8 \mathrm{~V}$ separately. The output is lower slightly owing to the electromagnetic damping in the composite output state, but the total power is $3.3 \mathrm{~mW}$ which is increased than single mode. The photo is shown in the Fig.9.

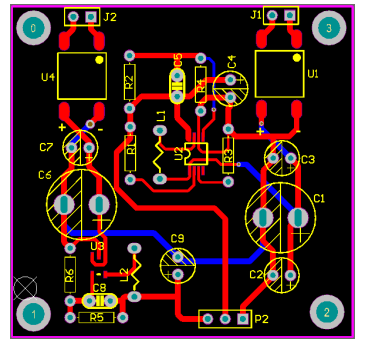

Fig.7 PCB board layout



Fig.8 Breadboard

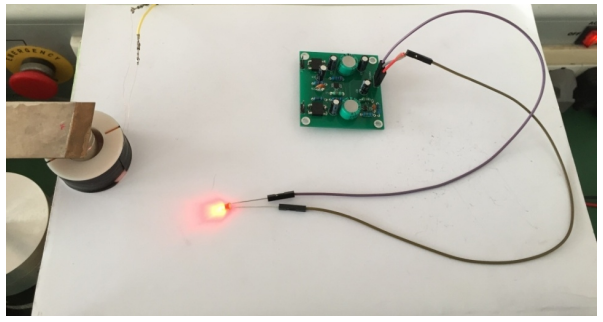

Fig.9 Photo of the experiment

\section{Summary}

In this paper, a feasible electromagnetic - piezoelectric composite vibration energy harvesting system is designed, and the feasibility and basic principle of the electromagnetic and piezoelectric energy harvesting are analyzed simply. An electromagnetic - piezoelectric composite vibration energy harvesting circuit is designed and we verify the composite energy harvesting system can get more energy than the single energy harvesting which could power the micro electromechanical devices successfully.

\section{ACKNOWLEDGEMENT}

This work was supported by the National Nature Science Foundation of China (No. 51307070), and the science and technology support of Huaiyin Institute of Technology.

\section{References}

[1] Yutaka Tomimatsu, Hidetoshi Takahashi, Takeshi Kobayashi et al., A piezoelectric cantilever-type differential pressure sensor for a low standby power trigger switch, Journal of Micromechanics and Microengineering. 23(2013)51-59.

[2] Khaligh A, Zeng P, Zheng C, Kinetic energy harvesting using piezoelectric and electromagnetic technologies - state of the art, IEEE Transactions on Industrial Electronics. 57(2010) 850-860.

[3] H.Kulah , K.Najafi , Energy scavenging from low-frequency vibrations by using frequency up-conversion for wireless sensor applications, IEEE Sensors Journal. 8(2008)261-268.

[4] Karol Waszczuk, Tomasz Piasecki, Karol Nitsch et al., Modulation of excitation signal as a 
method for measurement of the mechanical properties of miniature piezoelectric resonant devices in a viscous and conductive environment, Measurement Science and Technology, 24(2013)1-7. 